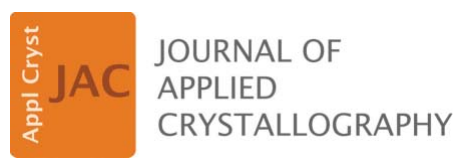

ISSN 1600-5767

Received 9 October 2018

Accepted 21 January 2019

Edited by G. Renaud, CEA-Grenoble DSM/ INAC/SP2M/NRS, Grenoble, France

Keywords: small-angle X-ray scattering; SAXS; absolute intensity; Au nanoparticle suspensions; reacting liquid jets; extended Guinier approximation.

Supporting information: this article has supporting information at journals.iucr.org/j

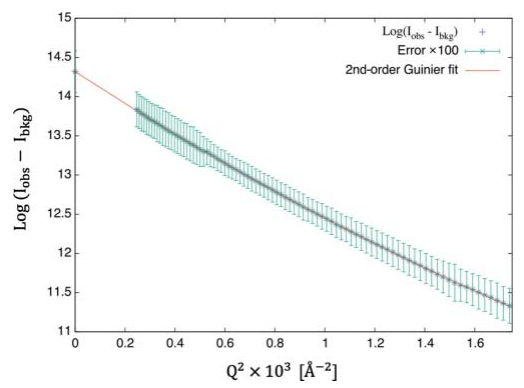

(C) 2019 International Union of Crystallography

\section{A dilute gold nanoparticle suspension as small-angle X-ray scattering standard for an absolute scale using an extended Guinier approximation}

\author{
Ahmed S. A. Mohammed, ${ }^{\mathrm{a}, \mathrm{b}, \mathrm{c}}$ Agnese Carino, ${ }^{\mathrm{d}}$ Andrea Testino, ${ }^{\mathrm{d}}$ Mohammad Reza \\ Andalibi $^{\mathrm{d}}$ and Antonio Cervellino ${ }^{\mathrm{a} *}$

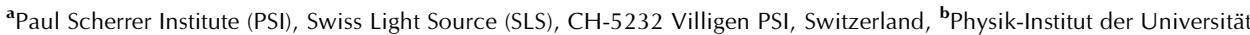 \\ Zürich, Winterthurerstrasse 190, CH-8057 Zürich, Switzerland, 'Physics Department, Faculty of Science, Fayoum \\ University, 63514 Fayoum, Egypt, and ${ }^{\mathbf{d}}$ Paul Scherrer Institute (PSI), ENE, CH-5232 Villigen PSI, Switzerland. \\ *Correspondence e-mail: antonio.cervellino@psi.ch
}

In this article, a practical procedure for absolute intensity calibration for smallangle scattering (SAXS) studies on liquid microjets is established. A gold nanoparticle suspension is used as standard so that the intercept at $Q=0$ of the SAXS scattering curve provides a scaling reference. In order to obtain the most precise extrapolation at $Q=0$, an extension of the Guinier approximation has been used, with a second-order term in the fit that adapts to a larger $Q$ range.

\section{Introduction}

We have been conducting a number of small-angle X-ray scattering (SAXS) experiments on the early stages of nucleation of simple mineral salts, such as calcium carbonate (Mohammed et al., 2019). The ideal setup was identified as a liquid jet containing the nucleating material. In order to extract the concentration of the nucleating entities at the time of measurement as seen by SAXS, given the impossibility of measuring it offline as the samples were in a dynamical state, an absolute intensity scale was needed for correlating the measured intensity to the number density of clusters contributing to the SAXS signal. This is the same method as reported by Hendricks et al. (1974) to quantify voids in metals. We have therefore been seeking a means of precisely quantifying a scale factor for bringing the SAXS signal onto an absolute scale. Different methods have been used in the past for this purpose (Pauw, 2013; Dreiss et al., 2006). We classify these broadly into three categories:

(a) Direct methods, i.e. measuring the direct beam intensity. The setup described by Zemb et al. (2003), where the direct beam is measured simultaneously by the use of a transparent beamstop of calibrated transmission, and other methods employing variable calibrated attenuators (Weinberg, 1963) fall into this category. As an example of a modern synchrotron beamline where absolute intensities can be obtained routinely owing to precise instrument calibration, we cite the ultrasmall-angle X-ray scattering beamline of the Advanced Photon Source (Ilavsky et al., 2009).

(b) Primary standards, namely a suitably chosen pure and homogeneous material whose SAXS signal can be calculated theoretically. The standard is measured in the same experimental conditions as the sample and then, by comparing theoretical and measured signals, a scale factor can be extracted. Such standards include gases (Weinberg, 1963), water and other pure liquids (Dingenouts \& Ballau, 1999; 
Orthaber et al., 2000), and solids such as glassy carbon (Perret \& Ruland, 1972). The latter has been certified (Allen et al., 2017) and is available to the scientific community through NIST. A polymer (Lupolen) has also been used (Kratky, 1964; Kratky et al., 1966; Russell et al., 1988). Incoherent scattering of vanadium for neutron scattering experiments (Hendricks $e t$ al., 1974) can also be classified here.

(c) Secondary standards, which are not pure homogeneous materials but rather two-phase (dilute) mixtures whose SAXS patterns reflect the SAXS scattering of the particles in suspension. An example is calibrated suspensions of monodisperse nanoparticles of silica (Patel \& Schmidt, 1971; Russell, 1983; Dreiss et al., 2006). In order to be used as absolute intensity standards, the particle suspensions need to be thoroughly characterized.

For our experiments with liquid jets, this wide portfolio of methods is considerably restricted. The beamline that we use here (X04SA-MS, Swiss Light Source synchrotron) is not dedicated to SAXS experiments, being instead a very versatile instrument (Willmott et al., 2013) with a broad continuous range of wavelengths $(0.4-2.5 \AA)$ and complex optics and beam-conditioning stages, with the accent on flexibility of operation. Therefore, instrument precalibration or direct methods were excluded as too costly in terms of time and resources or downright unfeasible. The necessity of maintaining exactly the same geometric setup, a quite strict requirement, rules out also the choice of standards that are not suitable to be fed through the liquid jet, such as gaseous or solid standards. Water - widely used as a primary SAXS standard - would be a possible choice, but for several reasons discussed in the following it is suboptimal. Instead, we developed a novel secondary SAXS standard for absolute intensity in order to derive the absolute scale constant from the extrapolation of the SAXS intensity for $Q \rightarrow 0$. This standard takes the form of a dilute suspension of heavy metal nanoparticles of which all necessary information is known and from which a predictable SAXS signal could be obtained in exactly the same configuration as our experiments. There are several nanoparticle systems that can be obtained as water suspensions, that are chemically stable over long periods, whose size and shape distribution is particularly simple (close to monodisperse spheres), and that can be separately characterized with good precision and little effort in a well equipped laboratory. Noble metal nanoparticles have obviously a superior SAXS contrast. Following this path, we identified as a suitable standard a dilute suspension of known concentration $\left(200 \mathrm{mg} \mathrm{l}^{-1}\right)$ of nearly spherical and monodisperse $18 \mathrm{~nm}$ citrate-stabilized $\mathrm{Au}$ nanoparticles. The extrapolation to $Q \rightarrow 0$ was achieved using an extension of the Guinier method. In particular, in order to use an extended data range, the classical Guinier approximation was extended to one more term (i.e. to the fourth order in $Q$ ).

\section{Liquid-microjet setup}

A free-liquid-jet setup was developed to collect in situ synchrotron small- and wide-angle X-ray scattering data
(Mohammed et al., 2017). Among different applications, this approach enables the study of the early stage of solid formation (nucleation). Such a mechanism is delicate and it is prone to be influenced by many factors (Carino et al., 2017; Marmiroli et al., 2009); in this field, in situ techniques are well established and are able to provide reliable experimental results (Haberkorn et al., 2003). A successful SAXS measurement setup was demonstrated by Schmidt et al. (2010) for $\mathrm{ZnS}$ precipitation. Here the aim is to study by in situ SAXS measurements very diluted inorganic systems containing entities with an electron density slightly higher than that of the solvent (water). Our study was specifically designed to investigate the formation pathway of biominerals, such as calcium carbonate and calcium phosphate, under controlled temperature, $\mathrm{pH}$ and saturation conditions. The pulsation-free horizontal-reacting liquid-jet setup is composed of four highpressure/high-performance liquid chromatography (HPLC) pumps (each of them equipped with a pulsation damper system and a high-precision Coriolis mass flowmeter), a micromixing system, a delay loop and a catcher. The micromixer manifold is equipped with five inputs and one output. Four inputs are connected to the solutions contributing to the precipitation reaction, whereas the fifth input was used for cleaning purposes and connected to an additional pump delivering $10 \mathrm{wt} \%$ acetic acid. The delay loop (which consists of a Teflon tube of a certain length and internal diameter) can be connected to the micromixer output. This tube determines the delay time between the mixing point and the measuring point, where the free-liquid jet is interrogated by the X-ray beam. A double-walled water-jacketed tubing system is used to thermostat the delay tube and the chemical delivery lines before the mixing point. Nozzles of different materials and sizes may be connected after the delay loop and used to generate the liquid jet. In this study, a stainless steel capillary with an internal diameter of $250 \mu \mathrm{m}$ and an overall flow rate of $8 \mathrm{ml} \mathrm{min}{ }^{-1}$ was used. The main advantage of this approach is that the solid formation mechanisms can be followed while the liquid jet is continuously refreshed, in a defined time frame after the mixing point. Therefore, the measurement is performed in static conditions and with negligible perturbations of the jet probed by the beam (negligible beam damage). Since the amount and electron density of the material of interest is very low, an accurate experimental data collection needs to be carried out and data accumulated for a relatively long time at each experimental condition (static mode).

A 'blank' solution, as similar as possible to the nucleating one but without the nucleating component, was also measured each time. As the absorption coefficients of the blank and of the nucleating liquid do not differ appreciably at the concentrations (far below $0.1 \%$ in mass) that are necessary for these studies, the blank provides a comprehensive background to be subtracted from the sample patterns. This background includes the scattering of the carrier liquid and that of air, another necessary evil when using the microjet setup.

The resulting difference signal can then be analysed to retrieve the size distribution of the prenucleation clusters. Moreover, in order to derive the concentration of these 
scattering objects (whose composition can be inferred from a kinetic model), the absolute scale constant needs to be evaluated. To this end, a well characterized diluted standard solution containing gold nanoparticles was used within exactly the same experimental configuration as the precipitation experiments, including a similar background subtraction procedure.

\section{Standard preparation and characterization}

The gold nanoparticle (GNP) suspension was synthesized using the citrate route, also called the Turkevich method [i.e. by reducing tetrachloroauric acid (TCAA) precursors with trisodium citrate $\left(\mathrm{Na}_{3} \mathrm{cit}\right)$ in water]. The preparation was done using a segmented-flow tubular reactor, SFTR (Jongen et al., 2003; Donnet et al., 2000; Aimable et al., 2011). The SFTR allows accurate monitoring of the ratio of the chemicals, the temperature and the ageing time. The segmentation is obtained using a secondary immiscible fluid which also prevents fouling. The precursor solutions are mixed at room temperature and segmented into the tubular reactor as a sequence of droplets in which the reaction occurs, triggered by the fast temperature rise of the tubular reactor. The obtained nanoparticle size distribution is narrow and reproducible. This reactor allows a continuous production of up to $5 \mathrm{~g}$ of solid per day. The conditions used to prepare the GNP suspension were similar to those reported in a previous study (Carino et al., 2016). The synthesis was done at $368 \mathrm{~K}$ using an $\mathrm{Na}_{3}$ cit to TCAA molar ratio equal to 7.9, an ageing time of $5 \mathrm{~min}$ and a chemical flow rate of $1 \mathrm{l} \mathrm{h}^{-1}$. The final GNP concentration was about $0.2 \mathrm{~g} \mathrm{l}^{-1}$ (measured by inductively coupled plasma mass spectrometry). The suspension exhibited a very narrow monomodal distribution of GNPs with a number-averaged diameter of $17.9 \mathrm{~nm}$ and a distribution r.m.s. width of $1.68 \mathrm{~nm}$, below $10 \%$ as measured by transmission electron microscopy (TEM) imaging and dynamic light scattering (DLS). TEM data are shown in Fig. 1, and DLS and additional disc centrifuge data (DC24000UHR, CPS Instruments Europe) are given in the supplementary information.

\section{Data collection}

Synchrotron SAXS measurements were carried out at the Materials Science beamline (X04SA-MS) of the Swiss Light Source at the Paul Scherrer Institute (Willmott et al., 2013). This synchrotron station is built mainly for wide-angle $\mathrm{X}$-ray scattering powder diffraction measurements but it also has some SAXS capabilities. The liquid jet, horizontal and orthogonal to the X-ray beam, was mounted on a double micrometric translation stage and optically centred with respect to the diffractometer circle by a high-resolu- tion camera. The X-ray beam was set at $9.5 \mathrm{keV}$ (corresponding to a wavelength $\lambda=1.305 \AA$ ), where the X-ray flux is maximal. Data were collected with the Mythen II detector system (Bergamaschi et al., 2010), which with its $0.0036^{\circ}$ step has a sufficient resolution for SAXS on this system. The minimum accessible $2 \theta$ scattering angle is $\sim 0.18^{\circ}$, corresponding to a minimum accessible momentum transfer of $Q=$ $4 \pi \sin (\theta) / \lambda=0.015 \AA^{-1}$. Scattering patterns were collected with reasonable acquisition times $(20 \mathrm{~min})$. Before the analysis, the experimental data were processed, corrected and rebinned to a uniform-step $Q$ grid, each data point being a triple $Q$-intensity-standard deviation.

\subsection{Extended Guinier approximation}

The Guinier approximation is a universal method (Guinier et al., 1955) to derive some fundamental parameters from SAXS data exploiting characteristic behaviour for $Q \rightarrow 0$ of the scattered intensity for diluted systems. If $I(Q)$ is the sample's scattered intensity, already background subtracted and corrected for all instrumental aberrations, the Guinier approximation is

$$
I(Q) \simeq A_{0} \exp \left(-b Q^{2}\right),
$$

where $A_{0}=I(Q=0)$ and $b=R_{\mathrm{g}}^{2} / 3$ is related to the sample (appropriately averaged) gyration radius [see Section A1, equation (13)]. This form is valid because $I(Q)$ is a smooth positive even function with a maximum at $Q=0$ and monotonically decreasing for sufficiently small $Q$. It is usually recast as its MacLaurin expansion:

$$
\log [I(Q)] \simeq \log \left(A_{0}\right)-b Q^{2} .
$$

A line fit of $\log [I(Q)]$ versus $Q^{2}$ then easily yields the values of $A_{0}$ and $b$. The range of validity of this approximation is, however, restricted to about

$$
Q<\pi / D
$$


where $D$ is the scattering particle's diameter (in the case of a sphere).

A straightforward extension of this approximation is possible, where we suppose that $I(Q)$ at small $Q$ can be described in the form

$$
I(Q) \simeq \exp \left(a-b Q^{2}+C Q^{4}\right),
$$

that is, with a further quartic term in the exponent. Again we perform the MacLaurin expansion,

$$
\log [I(Q)] \simeq \log \left(A_{0}\right)-b Q^{2}+c Q^{4}, \quad c \equiv C+b^{2} / 2,
$$

where a second-order polynomial in $Q^{2}$ replaces the straight line. A more technical derivation is given in Appendix $A$.

\subsection{Determination of the scale factor}

We employ a water suspension of spherical $\mathrm{Au}$ nanoparticles of known concentration $\left(200 \mathrm{mg} \mathrm{l}^{-1}\right)$ and known, practically unimodal size $(18 \mathrm{~nm})$ as standard to determine the absolute scale factor $k$. Such a suspension can be measured with the liquid jet with exactly the same setup as the investigated samples in this project. The observed intensity per unit time (after subtracting the background, including that of the liquid, measured separately) is

$$
I(Q)=k N(\Delta \rho)^{2} V_{\mathrm{p}}^{2}\left[3 \frac{\sin (Q R)-Q R \cos (Q R)}{(Q R)^{3}}\right]^{2},
$$

where $k$ is a constant pertaining to the experimental setup, $N$ is the number of illuminated particles, $\Delta \rho$ is the particles' scattering contrast (the difference in scattering length density with respect to the average; hereafter we use electron units, where the classical electron radius $r_{\mathrm{e}}=1$, so $\Delta \rho$ has units of inverse volume), $V_{\mathrm{p}}$ is the particle volume and the term within square brackets is the analytical expression of the scattering intensity of a sphere.

The SAXS patterns were analysed using the extended Guinier approximation of equation (5), discussed in Section 4.1. The parabolic fit is shown in Fig. 2. As our $Q$ range contains relatively few points within the strict limits of validity of the standard Guinier approximation, the quadratic term in the fit allowed us to include an about three times wider $Q$ range, up to $Q \simeq 6 / D$. The significance of the second-order term is immaterial within the scope of this paper. It helps us to obtain a more precise estimate of the other two parameters, especially $A_{0}$. The extended Guinier fit yielded a gyration radius of $7.88 \pm 0.012 \mathrm{~nm}$, corresponding - in the case of perfectly monodisperse spherical particles - to a particle diameter of $20.35 \pm 0.03 \mathrm{~nm}$. This value is $13 \%$ higher than the TEM-estimated number-averaged diameter of $17.9 \mathrm{~nm}$. However, our SAXS intercept-derived value is a different quantity from the TEM value. The latter is a visual number average over a few hundred imaged particles. As such, it can suffer from poor statistics due to the limited number of particles. On the other side, the Guinier intercept method averages the gyration radius (and not the actual diameter) over the squared-mass distribution. It has been shown that this leads to values consistently higher than the number-averaged diameter, with the overshoot depending on the distribution r.m.s. width (Mori et al., 2006). So the expected Guinier value for the TEM distribution (r.m.s. width $1.69 \mathrm{~nm}$ ) would be about $19 \mathrm{~nm}$. Further similar contributions due $(a)$ to (very likely) asphericity of the GNPs - leading to a larger gyration radius for the same diameter - and $(b)$ to the presence of a $\sim 0.5 \mathrm{~nm}$ layer of surfactant (citrate) can be expected. These corrections make the Guinier size compatible with values obtained by other methods. As the Guinier-derived diameter is the most appropriate for calculating the particle volume to be used in equation (6), and it is obtained simultaneously with the intercept from the same fit, a small polydispersity of the standard does not affect the precision of the method.

Finally, the extrapolated forward intensity was found to be

$$
A_{0}=I(Q=0)=1.653 \times 10^{6} \pm 4 \times 10^{3} \text { counts per second }
$$

The average electron density of the suspension can be approximated with that of pure water, given the low concentration. For the GNPs the density of bulk gold will be used. Therefore, evaluating the electron densities yields $\rho_{\mathrm{H}_{2} \mathrm{O}}=$ $3.346 \times 10_{23} \mathrm{~cm}^{-3}, \rho_{\mathrm{Au}}=4.661 \times 10^{24} \mathrm{~cm}^{-3}$ and $\Delta \rho=4.326 \times$ $10^{24} \mathrm{~cm}^{-3}$. From the given concentration and the Guinier diameter, we can assess that the number of GNPs per cubic centimetre is $n=2.350 \times 10^{12} \mathrm{~cm}^{-3}$, and as the illuminated liquid volume was calculated as $V=1.964 \times 10^{-4} \mathrm{~cm}^{3}$, we have a total of $N=n V=4.614 \times 10^{8}$ illuminated GNPs. The particle volume, from the estimated diameter of $20.346 \mathrm{~nm}$, results as $V_{\mathrm{p}}=4.410 \times 10^{-18} \mathrm{~cm}^{3}$. At $Q=0$, the term in square brackets in equation (6) is 1 , so we can now solve for the scale factor:

$$
k=\frac{I(Q=0)}{N(\Delta \rho)^{2} V_{\mathrm{p}}^{2}}=1.011 \times 10^{-17} .
$$

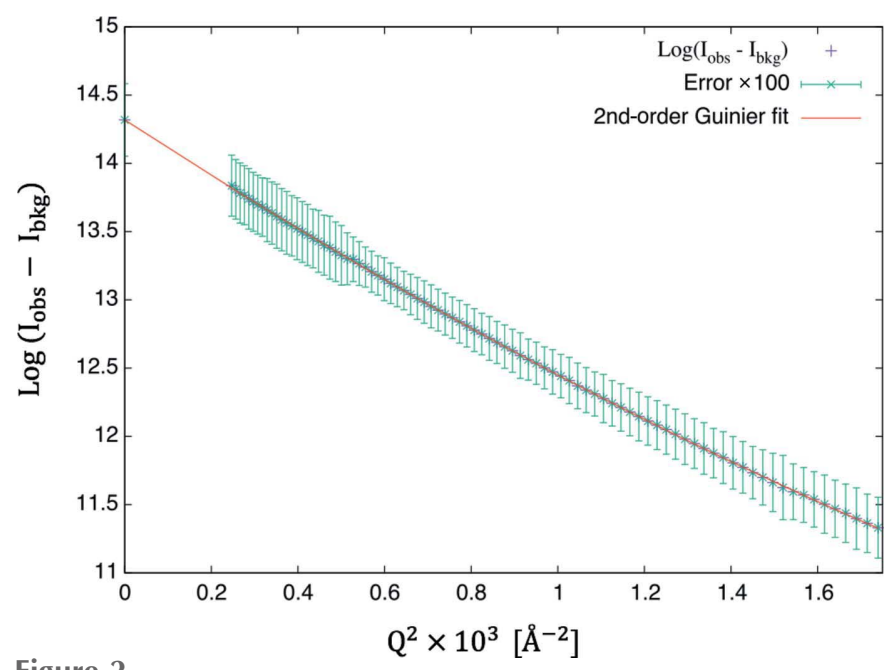

Figure 2

Guinier fit with second-order term added in order to exploit a larger $Q$ range and obtain a good estimate of the intercept, as needed for the absolute scale factor estimation. 


\subsection{Verification of the scale factor}

In order to test the effectiveness of our secondary standard, we have also run a comparison with the only other possible method from the literature, that is, using water as a primary standard. In passing, we mention that the only other obvious choice - a standard suspension of silica nanoparticles in water - had to be discarded. In fact, silica has a lower contrast relative to water (about seven times less compared with gold) and to obtain a comparable signal strength much higher concentrations have to be used [1-10\% volume as reported by Russell (1983)]. This entails a serious risk of clogging the very expensive apparatus producing the microjet, with no easy cleaning method at hand.

Water is very well suited to the liquid-jet device, built for aqueous liquids, but unfortunately it is suboptimal as a standard for two reasons. Firstly, the basic assumption that the water scattering is practically constant does not hold, as we observed, because it is true only within a rough level of approximation. Water patterns showed a $Q$ dependence in the experimental range and a certain lack of reproducibility, essentially due to the thermal variation of the isothermal compressibility and of the water structure factor. A great deal of interesting science, in fact, results from studying SAXS patterns of water (Huang et al., 2009; Clark et al., 2010; Gallo et al., 2016). Secondly, for the scattering pattern of the water jet the background is essentially that of air scattering. This was also measured separately, but then of course the air pattern must be corrected for absorption, which introduces a further error because absorption by the liquid jet affects only the air in the beam-path portion after the jet. However, we made a best possible estimate, resulting in a transmission factor of $0.89 \pm 0.01$ at a wavelength of $1.3 \AA$. The $Q$ range from 1 to 5 was relatively flat, so it was used for checking the scale factor. According to Orthaber et al. (2000), the value of the differential cross section per unit volume of water at $293 \mathrm{~K}$ is

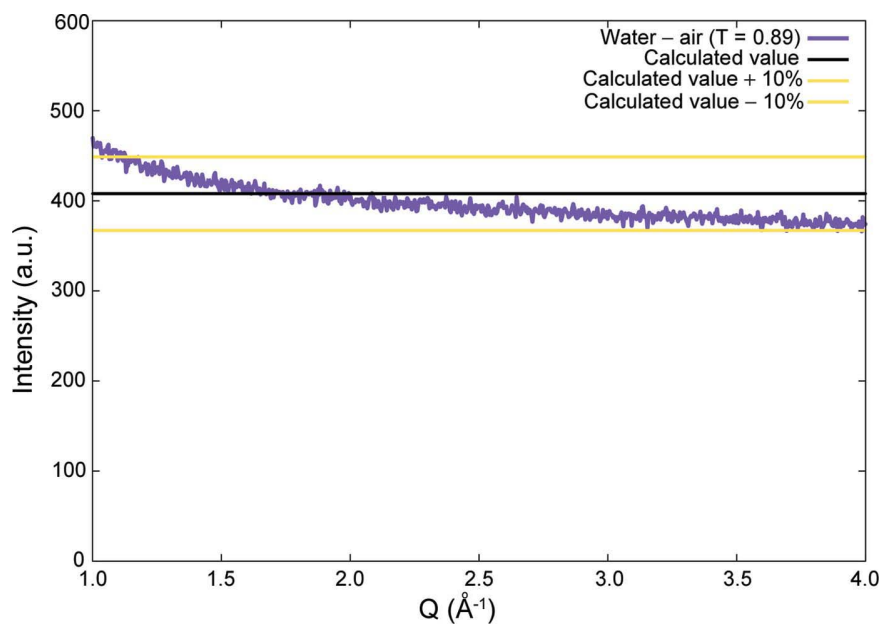

Figure 3

Pattern of water corrected by subtraction of the air scattering pattern and multiplied by the calculated transmission factor of 0.89 . Black line: value calculated from the constant cross section of water at $293 \mathrm{~K}$ with the scale factor obtained from the Au suspension. Yellow lines mark $\pm 10 \%$ of the calculated value.
$1.632 \times 10^{-2} \mathrm{~cm}^{-1}$. As we work in electron units (where the classical electron radius is 1 ), we have to divide this value by $r_{\mathrm{e}}^{2}=7.94 \times 10^{-26} \mathrm{~cm}^{2}$ and multiply it by the sample illuminated volume $V=1.964 \times 10^{-4} \mathrm{~cm}^{3}$. Multiplying also by the scale factor $k=1.011 \times 10^{-17}$, we obtain a value of 408 . The agreement (see Fig. 3) with the data is reasonable (within 10\% maximum) given that the best values from cited papers indicate a $5 \%$ error.

\section{Conclusions}

We have demonstrated an external standard method to evaluate the scale factor for absolute intensity in SAXS experiments involving a liquid jet. The standard for aqueous-liquidjet-type experiments is a suspension of nearly monodisperse nanoparticles of known concentration, high contrast, close to spherical shape and a known size that is similar to that of the samples under investigation (in order to obtain a good signal in the experimental $Q$ range without having to modify the setup). An extended Guinier approximation has been introduced, in order to improve the fit and consequently to achieve a more precise estimate of the extrapolated intensity at $Q=0$. The method is simple, cheap, rapid (as it requires only one additional scattering measurement), and easily applicable to any beamline or instrument where a liquid-jet device is installed. This also removes the need to perform a much tougher calibration of the instrument when absolute scale data are desired, while preserving the same flexibility of operation of relative-scale instruments. We have also compared this method with the classical method of water scattering, obtaining a good agreement.

\section{APPENDIX $A$}

\section{Theory}

The Guinier approximation can be more rigorously derived by using its universal expression given by the Debye scattering equation (free from interparticle interference effects, as appropriate for diluted systems). The Debye scattering equation (Debye, 1915) yields the orientation-averaged differential cross section directly from the atomic scattering density. Note that as we consider a very small $Q$ range, we can assume that atomic scattering factors (scattering lengths) are constant and independent of $Q$ for X-rays, slightly simplifying the discussion.

The atomic scattering density of a particle containing $N_{\mathrm{a}}$ atoms that have scattering lengths (or scattering factors) $b_{j}$ and are located at positions $\mathbf{r}_{j}$ for $j=1, \ldots, N_{\mathrm{a}}$ is represented as

$$
\rho(\mathbf{r})=\sum_{j=1}^{N_{\mathrm{a}}} b_{j} \delta\left(\mathbf{r}-\mathbf{r}_{j}\right) .
$$

As the origin is arbitrary, we refer the atomic coordinates to the scattering centre of mass, so that

$$
\sum_{j=1}^{N_{\mathrm{a}}} b_{j} \mathbf{r}_{j} \equiv 0 .
$$


We also call $B_{1}$ and $B_{2}$ the sum of scattering lengths and that of their squares, respectively:

$$
B_{1} \equiv \sum_{j=1}^{N_{\mathrm{a}}} b_{j}, \quad B_{2} \equiv \sum_{j=1}^{N_{\mathrm{a}}} b_{j}^{2} .
$$

\section{A1. Radial moments}

The radial moments of a density are defined as

$$
M_{n}=\frac{\int \mathrm{d}^{3} \mathbf{r} \rho(\mathbf{r}) \mathbf{r}^{n}}{\int \mathrm{d}^{3} \mathbf{r} \rho(\mathbf{r})},
$$

where $\mathbf{r}^{2 m} \equiv(\mathbf{r} \cdot \mathbf{r})^{m}=r^{2 m}$ for even $n$ and $\mathbf{r}^{2 m+1} \equiv(\mathbf{r} \cdot \mathbf{r})^{m} \mathbf{r}=$ $r^{2 m} \mathbf{r}$ for odd $n$. From equation (10), the odd moments are all zero. We can now evaluate the even radial moments of this density:

$$
M_{2 n}=\frac{\int \mathrm{d}^{3} \mathbf{r} \rho(\mathbf{r}) r^{2 n}}{\int \mathrm{d}^{3} \mathbf{r} \rho(\mathbf{r})}=\frac{1}{B_{1}} \sum_{j=1}^{N_{\mathrm{a}}} b_{j} r_{j}^{2 n} .
$$

In particular, $M_{2}$ is called the squared gyration radius:

$$
r_{\mathrm{g}}^{2}=M_{2}
$$

The differential cross section as a function of the momentum transfer $\mathbf{Q}$ (where $Q=4 \pi \sin \theta / \lambda$ ) is

$$
\begin{aligned}
I(\mathbf{Q}) & =\sum_{j, k=1}^{N_{\mathrm{a}}} b_{j} b_{k} \exp \left[i \mathbf{Q} \cdot\left(\mathbf{r}_{j}-\mathbf{r}_{k}\right)\right] \\
& =B_{2}+2 \sum_{j>k=1}^{N_{\mathrm{a}}} b_{j} b_{k} \exp \left[i \mathbf{Q} \cdot\left(\mathbf{r}_{j}-\mathbf{r}_{k}\right)\right] .
\end{aligned}
$$

The first term on the right-hand side is the self-scattering $B_{2}$ [cf. equation (11)]. Averaging over all possible $\mathbf{Q}$ directions, we obtain the Debye scattering equation [with $\operatorname{sinc}(x) \equiv \sin (x) / x]$ :

$$
\begin{aligned}
I(Q) & =\sum_{j, k=1}^{N_{\mathrm{a}}} b_{j} b_{k} \operatorname{sinc}\left(Q\left|\mathbf{r}_{j}-\mathbf{r}_{k}\right|\right) \\
& =B_{2}+2 \sum_{j>k=1}^{N_{\mathrm{a}}} b_{j} b_{k} \operatorname{sinc}\left(Q d_{j k}\right),
\end{aligned}
$$

where $d_{j k} \equiv\left|\mathbf{r}_{j}-\mathbf{r}_{k}\right|$. Now we consider only the structural term $I_{\mathrm{s}}(Q)=I(Q)-B_{2}$. The pair correlation function, that is the sinc transform of $I_{\mathrm{s}}(Q)$, is

$$
f_{\mathrm{s}}(r)=\int_{0}^{+\infty} 4 \pi Q^{2} \mathrm{~d} Q \operatorname{sinc}(Q r) I_{\mathrm{s}}(Q)=2 \sum_{j>k=1}^{N_{\mathrm{a}}} \frac{b_{j} b_{k}}{4 \pi r d_{j k}} \delta\left(r-d_{j k}\right) .
$$

We want to calculate

$$
\begin{aligned}
Z_{2} & =\int_{0}^{+\infty} 4 \pi r^{2} \mathrm{~d} r f_{\mathrm{s}}(r) r^{2}=2 \sum_{j>k=1}^{N_{\mathrm{a}}} b_{j} b_{k} d_{j k}^{2} \\
& =\sum_{j, k=1}^{N_{\mathrm{a}}} b_{j} b_{k}\left(r_{j}^{2}+r_{k}^{2}-2 \mathbf{r}_{j} \cdot \mathbf{r}_{k}\right),
\end{aligned}
$$

where we have expanded the distance squared and re-added the diagonal terms as they are zero anyway. Now we split the sum into three parts, each with one of the terms in brackets, and reorder:

$$
\begin{aligned}
\sum_{j, k=1}^{N_{\mathrm{a}}} b_{j} b_{k} r_{j}^{2} & =\sum_{j, k=1}^{N_{\mathrm{a}}} b_{j} b_{k} r_{k}^{2}=B_{1} \sum_{j=1}^{N_{\mathrm{a}}} b_{j} r_{j}^{2}=B_{1}^{2} r_{\mathrm{g}}^{2}-2 \sum_{j, k=1}^{N_{\mathrm{a}}} b_{j} b_{k} \mathbf{r}_{j} \cdot \mathbf{r}_{k} \\
& =-2\left(\sum_{k=1}^{N_{\mathrm{a}}} b_{k} \mathbf{r}_{k}\right) \cdot\left(\sum_{j=1}^{N_{\mathrm{a}}} b_{j} \mathbf{r}_{j}\right)=0 .
\end{aligned}
$$

The last equality is a consequence of equation (10). Therefore $Z_{2}=2 B_{1}^{2} r_{\mathrm{g}}^{2}$ and the gyration radius can be evaluated directly from the pair correlation function, as

$$
r_{\mathrm{g}}^{2}=Z_{2} / 2 B_{1}^{2}
$$

using equations (11) and (18).

The Guinier approximation can now be derived from equation (16). There we can expand $I(Q)$ for small $Q$ in a MacLaurin series:

$$
\begin{aligned}
I(Q)= & \sum_{j, k=1}^{N_{\mathrm{a}}} b_{j} b_{k}-\frac{Q^{2}}{6} \sum_{j, k=1}^{N_{\mathrm{a}}} b_{j} b_{k} d_{j k}^{2}+\frac{Q^{4}}{120} \sum_{j, k=1}^{N_{\mathrm{a}}} b_{j} b_{k} d_{j k}^{4} \\
& -\frac{Q^{6}}{5040} \sum_{j, k=1}^{N_{\mathrm{a}}} b_{j} b_{k} d_{j k}^{6}+\cdots .
\end{aligned}
$$

$I(Q)$ being a positive, continuous and even function, its logarithm can also be expanded in a MacLaurin series which will contain only even powers of $Q$. Therefore, if we equate

$$
I(Q)=\exp \left(\sum_{m=1}^{+\infty} a_{m} Q^{2 m}\right)
$$

and expand it also in a MacLaurin series, we can then equate coefficients of equal powers of $Q$. The first three terms (up to $Q^{4}$ ) yield three equations:

$$
\begin{gathered}
\exp \left(a_{0}\right)=\sum_{j, k=1}^{N_{\mathrm{a}}} b_{j} b_{k}=B_{1}^{2}, \\
a_{1} \exp \left(a_{0}\right)=\frac{1}{6} \sum_{j, k=1}^{N_{\mathrm{a}}} b_{j} b_{k} d_{j k}^{2}=-\frac{1}{3} B_{1}^{2} r_{\mathrm{g}}^{2}, \\
\frac{1}{2}\left(a_{1}^{2}+2 a_{2}\right) \exp \left(a_{0}\right)=\frac{1}{120} \sum_{j, k=1}^{N_{\mathrm{a}}} b_{j} b_{k} d_{j k}^{4},
\end{gathered}
$$

and so on. Solving the first two equations, we have

$$
a_{0}=2 \log \left(B_{1}\right), \quad a_{1}=-\frac{1}{3} B_{1}^{2} r_{\mathrm{g}}^{2}
$$

as in the classical Guinier approximation. From the third equation we could derive an expression for a higher radial moment $M_{4}$.

\section{Funding information}

Funding for this research was provided by Paul Scherrer Institut. 


\section{References}

Aimable, A., Jongen, N., Testino, A., Donnet, M., Lemaître, J., Hofmann, H. \& Bowen, P. (2011). Chem. Eng. Technol. 34, 344352.

Allen, A. J., Zhang, F., Kline, R. J., Guthrie, W. F. \& Ilavsky, J. (2017). J. Appl. Cryst. 50, 462-474.

Bergamaschi, A., Cervellino, A., Dinapoli, R., Gozzo, F., Henrich, B., Johnson, I., Kraft, P., Mozzanica, A., Schmitt, B. \& Shi, X. (2010). J. Synchrotron Rad. 17, 653-668.

Carino, A., Testino, A., Andalibi, M. R., Pilger, F., Bowen, P. \& Ludwig, C. (2017). Cryst. Growth Des. 17, 2006-2015.

Carino, A., Walter, A., Testino, A. \& Hofmann, H. (2016). CHIMIA Int. J. Chem. 70, 457.

Clark, G. N. I., Hura, G. L., Teixeira, J., Soper, A. K. \& Head-Gordon, T. (2010). Proc. Natl Acad. Sci. USA, 107, 14003-14007.

Debye, P. (1915). Ann. Phys. 351, 809-823.

Dingenouts, N. J. B. P. D. \& Ballau, M. (1999). Adv. Polym. Sci. 144, 1-47.

Donnet, M., Jongen, N., Lemaître, J. \& Bowen, P. (2000). J. Mater. Sci. Lett. 19, 749-750.

Dreiss, C. A., Jack, K. S. \& Parker, A. P. (2006). J. Appl. Cryst. 39, 3238.

Gallo, P., Amann-Winkel, K., Angell, C. A., Anisimov, M. A., Caupin, F., Chakravarty, C., Lascaris, E., Loerting, T., Panagiotopoulos, A. Z., Russo, J., Sellberg, J. A., Stanley, H. E., Tanaka, H., Vega, C., Xu, L. \& Pettersson, L. G. M. (2016). Chem. Rev. 116, 7463-7500.

Guinier, A., Fournet, G. \& Walker, C. (1955). Small Angle Scattering of $X$-rays. New York: J. Wiley \& Sons.

Haberkorn, H., Franke, D., Frechen, T., Goesele, W. \& Rieger, J. (2003). J. Colloid Interface Sci. 259, 112-126.

Hendricks, R., Schelten, J. \& Schmatz, W. (1974). Philos. Mag. 30, 819-837.

Huang, C., Wikfeldt, K. T., Tokushima, T., Nordlund, D., Harada, Y., Bergmann, U., Niebuhr, M., Weiss, T. M., Horikawa, Y., Leetmaa, M., Ljungberg, M. P., Takahashi, O., Lenz, A., Ojamae, L., Lyubartsev, A. P., Shin, S., Pettersson, L. G. M. \& Nilsson, A. (2009). Proc. Natl Acad. Sci. USA, 106, 15214-15218.

Ilavsky, J., Jemian, P. R., Allen, A. J., Zhang, F., Levine, L. E. \& Long, G. G. (2009). J. Appl. Cryst. 42, 469-479.
Jongen, N., Donnet, M., Bowen, P., Lemaître, J., Hofmann, H., Schenk, R., Hofmann, C., Aoun-Habbache, M., Guillemet-Fritsch, S., Sarrias, J., Rousset, A., Viviani, M., Buscaglia, M. T., Buscaglia, V., Nanni, P., Testino, A. \& Herguijuela, J. R. (2003). Chem. Eng. Technol. 26, 303-305.

Kratky, O. (1964). Z. Anal. Chem. 201, 161-194.

Kratky, O., Pilz, I. \& Schmitz, P. (1966). J. Colloid Interface Sci. 21, 24-34.

Marmiroli, B., Grenci, G., Cacho-Nerin, F., Sartori, B., Ferrari, E., Laggner, P., Businaro, L. \& Amenitsch, H. (2009). Lab Chip, 9, 2063-2069.

Mohammed, A., Carino, A., Testino, A., Andalibi, M. \& Cervellino, A. (2019). Unpublished.

Mohammed, A. S., Cervellino, A., Testino, A. \& Carino, A. (2017). Acta Cryst. A73, C315.

Mori, Y., Furukawa, M., Hayashi, T. \& Nakamura, K. (2006). Paticulate Sci. Technol. 24, 97-103.

Orthaber, D., Bergmann, A. \& Glatter, O. (2000). J. Appl. Cryst. 33, 218-225.

Patel, I. S. \& Schmidt, P. W. (1971). J. Appl. Cryst. 4, 50-55.

Pauw, B. (2013). J. Phys. Condens. Matter, 25, 383201.

Perret, R. \& Ruland, W. (1972). J. Appl. Cryst. 5, 116-119.

Russell, T. P. (1983). J. Appl. Cryst. 16, 473-478.

Russell, T. P., Lin, J. S., Spooner, S. \& Wignall, G. D. (1988). J. Appl. Cryst. 21, 629-638.

Schmidt, W., Bussian, P., Lindén, M., Amenitsch, H., Agren, P., Tiemann, M. \& Schüth, F. (2010). J. Am. Chem. Soc. 132, 68226826.

Weinberg, D. (1963). Rev. Sci. Instrum. 34, 691-696.

Willmott, P. R., Meister, D., Leake, S. J., Lange, M., Bergamaschi, A., Böge, M., Calvi, M., Cancellieri, C., Casati, N., Cervellino, A., Chen, Q., David, C., Flechsig, U., Gozzo, F., Henrich, B., JäggiSpielmann, S., Jakob, B., Kalichava, I., Karvinen, P., Krempasky, J., Lüdeke, A., Lüscher, R., Maag, S., Quitmann, C., Reinle-Schmitt, M. L., Schmidt, T., Schmitt, B., Streun, A., Vartiainen, I., Vitins, M., Wang, X. \& Wullschleger, R. (2013). J. Synchrotron Rad. 20, 667682.

Zemb, T., Tache, O., Né, F. \& Spalla, O. (2003). J. Appl. Cryst. 36, 800805. 